\title{
RICCI RECURRENT $C R$ SUBMANIFOLDS OF A COMPLEX SPACE FORM
}

\author{
By \\ Mayuko KoN
}

\begin{abstract}
We show that there is no $C R$ submanifold with semi-flat normal connection and with recurrent Ricci tensor in a complex space form of nonzero constant holomorphic sectional curvature, if the dimension of its holomorphic distribution is greater than 2 .
\end{abstract}

\section{Introduction}

There are many results about real hypersurfaces immersed in a complex space form with additional conditions for the curvature tensor and the Ricci tensor. In [7] Kon proved that there are no Einstein real hypersurfaces of a complex projective space $C P^{m}$ and determined connected complete pseudo-Einstein real hypersurfaces in $C P^{m}$ (see also Cecil and Ryan [1]). Moreover, Ki [4] proved the nonexistence of real hypersurfaces with parallel Ricci tensor of a nonflat complex space form.

If the Ricci tensor $S$ of a Riemannian manifold $M$ satisfies the condition $\nabla S=S \otimes \alpha$ for some 1 -form $\alpha$, then $M$ is said to be Ricci recurrent. In the theory of Ricci recurrent manifolds, Patterson proved some important formulas in [11] and [12], which are developed by Roter [13] and Olszak [10] and are useful for our theory.

Recently, Hamada [3] showed that there are no real hypersurfaces with recurrent Ricci tensor of $C P^{m}$ under the condition that the structure vector field $\xi$ of the real hypersurface is a principal curvature vector field. Moreover, Loo [8] proved the theorem above without the assumption that the structure vector field $\xi$ of the real hypersurface is a principal curvature vector field.

A submanifold $M$ of a Kählerian manifold $\tilde{M}$ is called a $C R$ submanifold of $\tilde{M}$ if there exists a differentiable distribution $H: x \rightarrow H_{x} \subset T_{x}(M)$ on $M$ sat-

2000 Mathematics Subject Classification. Primary 53C40, 53C55 Secondary 53C25.

Key words and phrases. CR submanifold, generic submanifold, complex space form, recurrent Ricci tensor, pseudo-Einstein real hypersurface.

Received February 6, 2006. 
isfying the conditions that $H$ is holomorphic, i.e., $J H_{x}=H_{x}$ for each $x \in M$, and the complementary orthogonal distribution $H^{\perp}: x \rightarrow H_{x}^{\perp} \subset T_{x}(M)$ is antiinvariant, i.e. $J H_{x}^{\perp} \subset T_{x}(M)^{\perp}$ for each $x \in M$.

Any real hypersurface of a Kählerian manifold is a $C R$ submanifold.

The main purpose of the present paper is to prove the following theorem.

THEOREM. Let $M$ be an n-dimensional $C R$ submanifold of a complex space form $M^{m}(c), c \neq 0$, with semi-flat normal connection. If $\operatorname{dim} H_{x}>2$, then $M$ is never Ricci recurrent.

In section 2, we prepare some definitions and basic formulas for $C R$ submanifolds of a complex space form $M^{m}(c)$. In section 3, we give an equation about the Ricci tensor of a $C R$ submanifold with semi-flat normal connection of a complex space form. In section 4, we give a useful proof of a proposition about a Ricci recurrent manifold in Olszak [10] for our calculation of a Ricci recurrent $C R$ submanifold with semi-flat normal connection. Combining this with the equation given in section 3, we prove our main theorem. In the last section, we give a characterization of pseudo-Einstein real hypersurfaces of complex space forms using the results of section 3 .

\section{Preliminaries}

Let $M^{m}(c)$ denote the complex space form of complex dimension $m$ (real dimension $2 m$ ) with constant holomorphic sectional curvature $4 c$. We denote by $J$ the almost complex structure of $M^{m}(c)$. The Hermitian metric of $M^{m}(c)$ is denoted by $G$.

Let $M$ be a real $n$-dimensional Riemannian manifold isometrically immersed in $M^{m}(c)$. We denote by $g$ the Riemannian metric induced on $M$ from $G$, and by $p$ the codimension of $M$, that is, $p=2 m-n$.

We denote by $T_{x}(M)$ and $T_{x}(M)^{\perp}$ the tangent space and the normal space of $M$ respectively.

Definition. A submanifold $M$ of a Kählerian manifold $\tilde{M}$ is called a $C R$ submanifold of $\tilde{M}$ if there exists a differentiable distribution $H: x \rightarrow H_{x} \subset T_{x}(M)$ on $M$ satisfying the following conditions:

(i) $H$ is holomorphic, i.e., $J H_{x}=H_{x}$ for each $x \in M$, and

(ii) the complementary orthogonal distribution $H^{\perp}: x \rightarrow H_{x}^{\perp} \subset T_{x}(M)$ is anti-invariant, i.e. $J H_{x}^{\perp} \subset T_{x}(M)^{\perp}$ for each $x \in M$. 
If $J T_{x}(M)^{\perp} \subset T_{x}(M)$ for any point $x$ of $M$, then we call $M$ a generic submanifold of $\tilde{M}$. Any real hypersurface of $\tilde{M}$ is obviously a generic submanifold of $\tilde{M}$.

In the following, we put $\operatorname{dim} H_{x}=h, \operatorname{dim} H_{x}^{\perp}=q$ and codimension $M=p$. If $q=0$ (resp. $h=0$ ) for any $x \in M$, then the $C R$ submanifold $M$ is a holomorphic submanifold (resp. anti-invariant submanifold or totally real submanifold) of $\tilde{M}$. If $p=q$ for any $x \in M$, then the $C R$ submanifold $M$ is a generic submanifold of $\tilde{M}$ (see [15]).

We denote by $\tilde{\nabla}$ the covariant differentiation in $M^{m}(c)$, and by $\nabla$ the one in $M$ determined by the induced metric. Then the Gauss and Weingarten formulas are given respectively by

$$
\tilde{\nabla}_{X} Y=\nabla_{X} Y+B(X, Y), \quad \tilde{\nabla}_{X} V=-A_{V} X+D_{X} V,
$$

for any vector fields $X$ and $Y$ tangent to $M$ and any vector field $V$ normal to $M$, where $D$ denotes the covariant differentiation with respect to the linear connection induced in the normal bundle $T(M)^{\perp}$ of $M$. We call both $A$ and $B$ the second fundamental form of $M$ and are related by $G(B(X, Y), V)=g\left(A_{V} X, Y\right)$. The second fundamental form $A$ and $B$ are symmetric. $A_{V}$ can be considered as a $(n, n)$-matrix.

The covariant derivative $\left(\nabla_{X} A\right)_{V} Y$ of $A$ is defined to be

$$
\left(\nabla_{X} A\right)_{V} Y=\nabla_{X}\left(A_{V} Y\right)-A_{D_{X} V} Y-A_{V} \nabla_{X} Y .
$$

If $\left(\nabla_{X} A\right)_{V} Y=0$ for any vector fields $X$ and $Y$ tangent to $M$, then the second fundamental form of $M$ is said to be parallel in the direction of the normal vector $V$. If the second fundamental form is parallel in any direction, it is said to be parallel. A vector field $V$ normal to $M$ is said to be parallel if $D_{X} V=0$ for any vector field $X$ tangent to $M$.

In the sequel, we assume that $M$ is a $C R$ submanifold of $M^{m}(c)$. The tangent space $T_{x}(M)$ of $M$ is decomposed as $T_{x}(M)=H_{x}+H_{x}^{\perp}$ at each point $x$ of $M$, where $H_{x}^{\perp}$ denotes the orthogonal complement of $H_{x}$ in $T_{x}(M)$. Similarly, we see that $T_{x}(M)^{\perp}=J H_{x}^{\perp}+N_{x}$, where $N_{x}$ is the orthogonal complement of $J H_{x}^{\perp}$ in $T_{x}(M)^{\perp}$.

For any vector field $X$ tangent to $M$, we put

$$
J X=P X+F X
$$

where $P X$ is the tangential part of $J X$ and $F X$ the normal part of $J X$. Then $P$ is an endomorphism on the tangent bundle $T(M)$ and $F$ is a normal bundle valued 1-form on the tangent bundle $T(M)$. 
For any vector field $V$ normal to $M$, we put

$$
J V=t V+f V,
$$

where $t V$ is the tangential part of $J V$ and $f V$ the normal part of $J V$. Then we see that $F P=0, f F=0, t F=0$ and $P t=0$.

We define the covariant derivatives of $P, F, t$ and $f$ by $\left(\nabla_{X} P\right) Y=\nabla_{X}(P Y)-$ $P \nabla_{X} Y,\left(\nabla_{X} F\right) Y=D_{X}(F Y)-F \nabla_{X} Y,\left(\nabla_{X} t\right) V=\nabla_{X}(t V)-t D_{X} V$ and $\left(\nabla_{X} f\right) V=$ $D_{X}(f V)-f D_{X} V$ respectively. We then have

$$
\begin{gathered}
\left(\nabla_{X} P\right) Y=A_{F Y} X+t B(X, Y), \\
\left(\nabla_{X} F\right) Y=-B(X, P Y)+f B(X, Y), \\
\left(\nabla_{X} t\right) V=-P A_{V} X+A_{f V} X, \\
\left(\nabla_{X} f\right) V=-F A_{V} X-B(X, t V) .
\end{gathered}
$$

For any vector fields $X$ and $Y$ in $H_{x}^{\perp}=t T(M)^{\perp}$ we obtain

$$
A_{F X} Y=A_{F Y} X \text {. }
$$

We notice that $P^{3}+P=0$, and hence $P$ defines an $f$-structure on $M$ (see [14]).

We denote by $R$ the Riemannian curvature tensor field of $M$. Then the equation of Gauss is given by

$$
\begin{aligned}
R(X, Y) Z=c\{ & g(Y, Z) X-g(X, Z) Y+g(P Y, Z) P X \\
& -g(P X, Z) P Y-2 g(P X, Y) P Z\}+A_{B(Y, Z)} X-A_{B(X, Z)} Y,
\end{aligned}
$$

for any $X, Y$ and $Z$ tangent to $M$.

We denote by $S$ the Ricci tensor field of $M$. Then

$$
\begin{aligned}
g(S X, Y)= & (n-1) c g(X, Y)+3 c g(P X, P Y) \\
& +\sum_{a} \operatorname{Tr} A_{a} g\left(A_{a} X, Y\right)-\sum_{a} g\left(A_{a}^{2} X, Y\right),
\end{aligned}
$$

where $A_{a}$ is the second fundamental form in the direction of $v_{a},\left\{v_{1}, \ldots, v_{p}\right\}$ being an orthonormal frame for $T_{x}(M)^{\perp}$, and $\operatorname{Tr}$ denotes the trace of an operator. From this the scalar curvature $r$ of $M$ is given by

$$
r=(n-1) n c+3(n-p) c+\sum_{a}\left(\operatorname{Tr} A_{a}\right)^{2}-\sum_{a} \operatorname{Tr} A_{a}^{2},
$$

where $p$ is the codimension of $M$, that is, $p=2 m-n$. 
The equation of Codazzi of $M$ is given by

$$
\begin{aligned}
& g\left(\left(\nabla_{X} A\right)_{V} Y, Z\right)-g\left(\left(\nabla_{Y} A\right)_{V} X, Z\right) \\
& \quad=c\{g(Y, P Z) g(X, J V)-g(X, P Z) g(Y, J V)-2 g(X, P Y) g(Z, J V)\} .
\end{aligned}
$$

We define the curvature tensor $R^{\perp}$ of the normal bundle of $M$ by

$$
R^{\perp}(X, Y) V=D_{X} D_{Y} V-D_{Y} D_{X} V-D_{[X, Y]} V .
$$

Then we have the equation of Ricci

$$
\begin{aligned}
& G\left(R^{\perp}(X, Y) V, U\right)+g\left(\left[A_{U}, A_{V}\right] X, Y\right) \\
& \quad=c\{g(Y, J V) g(X, J U)-g(X, J V) g(Y, J U)-2 g(X, P Y) g(V, J U)\} .
\end{aligned}
$$

If $R^{\perp}$ vanishes identically, the normal connection of $M$ is said to be flat. We can see that the normal connection of $M$ is flat if and only if there exist locally $p$ mutually orthogonal unit normal vector fields $v_{a}$ such that each $v_{a}$ is parallel. If $R^{\perp}(X, Y) V=2 c g(X, P Y) f V$, then the normal connection of $M$ is said to be semi-flat (see [15]). The justification of this definition, see [15]. We notice that, if $M$ is a generic submanifold of $M^{m}(c)$, then $f$ vanishes identically, and hence $R^{\perp}=0$.

A nonzero tensor field $K$ of type $(r, s)$ on $M$ is said to be recurrent if there exists a 1 -form $\alpha$ such that $\nabla K=K \otimes \alpha . M$ is said to be Ricci recurrent if the Ricci tensor $S$ of $M$ is recurrent, that is, $S$ is nonzero and $\left(\nabla_{X} S\right) Y=\alpha(X) S Y$ for any vector fields $X$ and $Y$.

Any real hypersurface $M$ of $M^{m}(c)(m \geq 3, c \neq 0)$ is not Einstein. Therefore, the Ricci tensor $S$ of a real hypersurface $M$ of $M^{m}(c)(m \geq 3, c \neq 0)$ is nonzero (see [7], [9]).

\section{Ricci Tensor of $C R$ Submanifolds}

In this section, we give some results about the Ricci tensor of a $C R$ submanifolds of a complex space form $M^{m}(c)$.

THEOREM 3.1. Let $M$ be an n-dimensional $C R$ submanifold of a complex space form $M^{m}(c), c \neq 0, \operatorname{dim} H_{x}>2$, with semi-flat normal connection. Suppose that the curvature tensor $R$ and the Ricci tensor $S$ satisfy $g((R(X, Y) S) Z, W)=0$ for any tangent vectors $X, Y, Z, W \in H_{x}$. Then we have

$$
g(S X, Y)=\frac{1}{h}\left(r-\sum_{a=1}^{q} g\left(S t v_{a}, t v_{a}\right)\right) g(X, Y)
$$


for any vectors $X, Y \in H_{x}$, where $r$ denotes the scalar curvature of $M$ and $\left\{v_{1}, \ldots, v_{q}\right\}$ is an orthonormal basis of $\mathrm{JH}_{x}^{\perp}$.

Proof. Since $g((R(X, Y) S) Z, W)=0$ for any tangent vectors $X, Y, Z, W \in$ $H_{x}$, the first Bianchi identity gives

$$
g(R(X, Y) S Z+R(Y, Z) S X+R(Z, X) S Y, W)=0 .
$$

We take an orthonormal basis $\left\{e_{1}, \ldots, e_{h}, t v_{1}:=e_{h+1}, \ldots, t v_{q}:=e_{n}\right\}$ of $T_{x}(M)$, where $\left\{e_{1}, \ldots, e_{h}\right\}$ is an orthonormal basis of $H_{x}$ and $\left\{v_{1}, \ldots, v_{q}\right\}$ is an orthonormal basis of $J H_{x}^{\perp}$. Then we have

$$
g\left(\sum_{i=1}^{h} R\left(e_{i}, P e_{i}\right) S X+\sum_{i=1}^{h} R\left(P e_{i}, X\right) S e_{i}+\sum_{i=1}^{h} R\left(X, e_{i}\right) S P e_{i}, Y\right)=0 .
$$

Since $P t v_{a}=0$ for $a=1, \ldots, q$, we have

$$
g\left(\sum_{i=1}^{n} R\left(e_{i}, P e_{i}\right) S X+\sum_{i=1}^{n} R\left(P e_{i}, X\right) S e_{i}+\sum_{i=1}^{n} R\left(X, e_{i}\right) S P e_{i}, Y\right)=0 .
$$

Since we have

$$
g\left(\sum_{i=1}^{n} R\left(P e_{i}, X\right) S e_{i}, Y\right)=-g\left(\sum_{i=1}^{n} R\left(e_{i}, X\right) S P e_{i}, Y\right),
$$

it follows that

$$
\sum_{i=1}^{n} g\left(R\left(e_{i}, P e_{i}\right) S X, Y\right)=2 \sum_{i=1}^{n} g\left(R\left(e_{i}, X\right) S P e_{i}, Y\right) .
$$

On the other hand, by the equation of Gauss, we have

$$
\begin{aligned}
\sum_{i} g\left(R\left(e_{i}, P e_{i}\right) S X, Y\right)= & (-2 h-4) c g(P S X, Y)+\sum_{i} g\left(A_{B\left(P e_{i}, S X\right)} e_{i}, Y\right) \\
& -\sum_{i} g\left(A_{B\left(e_{i}, S X\right)} P e_{i}, Y\right) \\
2 \sum_{i} g\left(R\left(e_{i}, X\right) S P e_{i}, Y\right)=c\{ & -2 g(P S X, Y)+2 g(P S P X, P Y) \\
& \left.+4 g(P X, P S P Y)-2 \sum_{i} g\left(S P e_{i}, P e_{i}\right) g(P X, Y)\right\} \\
+ & 2 \sum_{i} g\left(A_{B\left(X, S P e_{i}\right)} e_{i}, Y\right)-2 \sum_{i} g\left(A_{B\left(e_{i}, S P e_{i}\right)} X, Y\right) .
\end{aligned}
$$


Thus we have

$$
\begin{aligned}
c\{(-2 h-2) g(P S X, Y)-2 g(P S P X, P Y)-4 g(P X, P S P Y)\} \\
=-2 c \sum_{i} g\left(S P e_{i}, P e_{i}\right) g(P X, Y)+2 \sum_{i, a} g\left(A_{a} e_{i}, Y\right) g\left(A_{a} X, S P e_{i}\right) \\
\quad-2 \sum_{i, a} g\left(A_{a} X, Y\right) g\left(A_{a} e_{i}, S P e_{i}\right)-2 \sum_{i, a} g\left(A_{a} e_{i}, Y\right) g\left(A_{a} P e_{i}, S X\right) .
\end{aligned}
$$

Since the Ricci tensor $S$ of $M$ is given by

$$
S X=(n-1) c X-3 c P^{2} X+\sum_{a} \operatorname{Tr} A_{a} \cdot A_{a} X-\sum_{a} A_{a}^{2} X
$$

we obtain, for $X, Y \in H_{x}$,

$$
\begin{aligned}
& \sum_{i, a} g\left(A_{a} e_{i}, Y\right) g\left(A_{a} X, S P e_{i}\right)-\sum_{i, a} g\left(A_{a} X, Y\right) g\left(A_{a} e_{i}, S P e_{i}\right) \\
&-\sum_{i, a} g\left(A_{a} e_{i}, Y\right) g\left(A_{a} P e_{i}, S X\right) \\
&= \sum_{i, a, b} \operatorname{Tr} A_{b} g\left(A_{a} e_{i}, Y\right) g\left(A_{a} X, A_{b} P e_{i}\right)-\sum_{i, a, b} g\left(A_{a} e_{i}, Y\right) g\left(A_{a} X, A_{b}^{2} P e_{i}\right) \\
&-\sum_{i, a, b} \operatorname{Tr} A_{b} g\left(A_{a} e_{i}, Y\right) g\left(A_{a} P e_{i}, A_{b} X\right)+\sum_{i, a, b} g\left(A_{a} e_{i}, Y\right) g\left(A_{a} P e_{i}, A_{b}^{2} X\right) \\
&-\sum_{i, a}(n-1) c g\left(A_{a} X, Y\right) g\left(A_{a} e_{i}, P e_{i}\right)+3 \sum_{i, a} c g\left(A_{a} X, Y\right) g\left(A_{a} e_{i}, P e_{i}\right) \\
&-\sum_{i, a, b} \operatorname{Tr} A_{b} g\left(A_{a} X, Y\right) g\left(A_{a} e_{i}, A_{b} P e_{i}\right)+\sum_{i, a, b} g\left(A_{a} X, Y\right) g\left(A_{a} e_{i}, A_{b}^{2} P e_{i}\right) \\
&=-\sum_{a, b} \operatorname{Tr} A_{b} g\left(A_{a} Y, P A_{b} A_{a} X\right)+\sum_{a, b} g\left(A_{a} Y, P A_{b}^{2} A_{a} X\right) \\
&+\sum_{a, b} \operatorname{Tr} A_{b} g\left(A_{a} Y, P A_{a} A_{b} X\right)-\sum_{a, b} g\left(A_{a} Y, P A_{a} A_{b}^{2} X\right) \\
&-\sum_{i, a, b} \operatorname{Tr} A_{b} g\left(A_{a} X, Y\right) g\left(A_{a} e_{i}, A_{b} P e_{i}\right)+\sum_{i, a, b} g\left(A_{a} X, Y\right) g\left(A_{a} e_{i}, A_{b}^{2} P e_{i}\right) .
\end{aligned}
$$

Since the normal connection of $M$ is semi-flat, the equation of Ricci gives

$$
A_{a} A_{b} X=A_{b} A_{a} X
$$


for any $X \in H_{x}$. Therefore, the equation above vanishes identically. From these equations and the assumption $c \neq 0$, we have

$$
(h+1) g(P S X, Y)+g(P S P X, P Y)+2 g(P X, P S P Y)=\sum_{i} g\left(S P e_{i}, P e_{i}\right) g(P X, Y),
$$

for any $X, Y \in H_{x}$. This implies

$$
(h-1) g(P S X, Y)+g(S P X, Y)=\sum_{i} g\left(S P e_{i}, P e_{i}\right) g(P X, Y) .
$$

Since $P X, P Y \in H_{x}$, we also have

$$
(h-1) g(P S P X, P Y)+g\left(S P^{2} X, P Y\right)=\sum_{i} g\left(S P e_{i}, P e_{i}\right) g(P X, Y),
$$

and hence

$$
(h-1) g(S P X, Y)+g(P S X, Y)=\sum_{i} g\left(S P e_{i}, P e_{i}\right) g(P X, Y) .
$$

From these equations, we obtain

$$
(h-2) g(S P X, P Y)=(h-2) g(S X, Y) .
$$

Since $h>2$, we have $g(S P X, P Y)=g(S X, Y)$. Thus, by the definition of the scalar curvature $r$ of $M$, we get

$$
\begin{aligned}
h g(S X, Y) & =\sum_{i} g\left(P S e_{i}, P e_{i}\right) g(X, Y) \\
& =\left(r-\sum_{a=1}^{q} g\left(S t v_{a}, t v_{a}\right)\right) g(X, Y),
\end{aligned}
$$

which proves our assertion.

When $M$ is a generic submanifold, the normal connection of $M$ is flat if $M$ is semi-flat. Let $p$ be the codimension of submanifold $M$ in $M^{m}(c)$ and $\left\{v_{1}, \ldots, v_{p}\right\}$ be an orthonormal basis of $T_{x}(M)^{\perp}$. Then we have the following theorem.

THEOREM 3.2. Let $M$ be an n-dimensional generic submanifold of a complex space form $M^{m}(c), c \neq 0, n-p>2$, with flat normal connection. Suppose that the 
curvature tensor $R$ and the Ricci tensor $S$ satisfy $g((R(X, Y) S) Z, W)=0$ for any tangent vectors $X, Y, Z, W \in H_{x}$. Then we have

$$
g(S X, Y)=\frac{1}{n-p}\left(r-\sum_{a=1}^{p} g\left(S J v_{a}, J v_{a}\right)\right) g(X, Y)
$$

for any vectors $X, Y \in H_{x}$.

Let $M$ be a real $(2 m-1)$-dimensional hypersurface immersed in $M^{m}(c)$. We take the unit normal vector field $N$ of $M$ in $M^{m}(c)$ and define a tangent vector field $\xi$ by $\xi=-J N$, which is called the structure vector field. As a corollary of Theorem 3.1, we have

COROLlary 3.3. Let $M$ be a real hypersurface of a complex space form $M^{m}(c), c \neq 0, m \geq 3$. Suppose that the curvature tensor $R$ and the Ricci tensor $S$ of $M$ satisfy $g((R(X, Y) S) Z, W)=0$ for any tangent vectors $X, Y, Z$ and $W$ orthogonal to $\xi$. Then we have

$$
g(S X, Y)=\frac{1}{2 m-2}(r-g(S \xi, \xi)) g(X, Y)
$$

for any tangent vectors $X$ and $Y$ orthogonal to $\xi$, where $r$ denotes the scalar curvature of $M$.

\section{Ricci Recurrent $C R$ Submanifolds}

In this section, we prove our main theorem. First, we give a useful proof of the proposition given by Olszak [10].

Proposition 4.1. Let $M$ be a Ricci recurrent manifold of dimension $n$ with $\alpha \neq 0$, where $\alpha$ is the recurrent form of the Ricci tensor. Then we have

$$
S^{2}=\frac{r}{2} S
$$

where $r$ denotes the scalar curvature of $M$.

Proof. By the definition of the Ricci recurrent manifold, the Ricci tensor $S$ of $M$ satisfies $\nabla S=S \otimes \alpha$. Then we have 


$$
\begin{aligned}
\left(\nabla_{X} \nabla_{Y} S\right) Z & =\left(\nabla_{X} \alpha\right)(Y) S Z+\alpha(Y)\left(\nabla_{X} S\right) Z+\alpha\left(\nabla_{X} Y\right) S Z \\
& =\left(\nabla_{X} \alpha\right)(Y) S Z+\alpha(Y) \alpha(X) S Z+\alpha\left(\nabla_{X} Y\right) S Z, \\
\left(\nabla_{Y} \nabla_{X} S\right) Z & =\left(\nabla_{Y} \alpha\right)(X) S Z+\alpha(X) \alpha(Y) S Z+\alpha\left(\nabla_{Y} X\right) S Z, \\
\left(\nabla_{[X, Y]} S\right) Z & =\alpha([X, Y]) S X .
\end{aligned}
$$

So we obtain

$$
(R(X, Y) S) Z=\left(\nabla_{X} \alpha\right)(Y) S Z-\left(\nabla_{Y} \alpha\right)(X) S Z .
$$

Since $S$ is symmetric and nonzero, we can choose some nonzero function $\lambda$ and a vector field $Z$ such that $S Z=\lambda Z$. Then

$$
(R(X, Y) S) Z=\lambda\left\{\left(\nabla_{X} \alpha\right)(Y) Z-\left(\nabla_{Y} \alpha\right)(X) Z\right\} .
$$

On the other hand, we have

$$
\begin{aligned}
g((R(X, Y) S) Z, Z) & =g(R(X, Y) S Z, Z)-g(S R(X, Y) Z, Z) \\
& =\lambda\{g(R(X, Y) Z, Z)-g(R(X, Y) Z, Z)\} \\
& =0
\end{aligned}
$$

Thus we obtain

$$
\left(\nabla_{X} \alpha\right)(Y)-\left(\nabla_{Y} \alpha\right)(X)=0
$$

By (4.1) and (4.2), we have $R(X, Y) S=0$. So we obtain, $R(X, Y) S Z$ $S R(X, Y) Z=0$, and hence

$$
\begin{aligned}
0 & =\left(\nabla_{W} R\right)(X, Y) S Z+R(X, Y)\left(\nabla_{W} S\right) Z-\left(\nabla_{W} S\right) R(X, Y) Z-S\left(\nabla_{W} R\right)(X, Y) Z \\
& =\left(\nabla_{W} R\right)(X, Y) S Z+\alpha(W) R(X, Y) S Z-\alpha(W) S R(X, Y) Z-S\left(\nabla_{W} R\right)(X, Y) Z \\
& =\left(\nabla_{W} R\right)(X, Y) S Z-S\left(\nabla_{W} R\right)(X, Y) Z .
\end{aligned}
$$

We take a basis $\left\{e_{1}, \ldots, e_{n}\right\}$ of $T_{x}(M)$. Generally we have

$$
\begin{aligned}
\sum_{i} g\left(\left(\nabla_{e_{i}} R\right)\left(e_{i}, X\right) Y, Z\right) & =\sum_{i} g\left(\left(\nabla_{e_{i}} R\right)(Z, Y) X, e_{i}\right) \\
& =-\sum_{i} g\left(\left(\nabla_{Z} R\right)\left(Y, e_{i}\right) X, e_{i}\right)-\sum_{i} g\left(\left(\nabla_{Y} R\right)\left(e_{i}, Z\right) X, e_{i}\right) \\
& =g\left(\left(\nabla_{Z} S\right) Y, X\right)-g\left(\left(\nabla_{Y} S\right) Z, X\right) .
\end{aligned}
$$


Using this, we obtain

$$
\begin{aligned}
0 & =\sum_{i}\left\{g\left(\left(\nabla_{e_{i}} R\right)\left(e_{i}, Y\right) S Z, X\right)-g\left(S\left(\nabla_{e_{i}} R\right)\left(e_{i}, Y\right) Z, X\right)\right\} \\
& =g\left(\left(\nabla_{X} S\right) S Z, Y\right)-g\left(\left(\nabla_{S Z} S\right) X, Y\right)-g\left(\left(\nabla_{S X} S\right) Z, Y\right)+g\left(\left(\nabla_{Z} S\right) S X, Y\right) \\
& =\alpha(X) g\left(S^{2} Z, Y\right)-\alpha(S X) g(S Z, Y)+\alpha(Z) g\left(S^{2} X, Y\right)-\alpha(S Z) g(S X, Y) .
\end{aligned}
$$

On the other hand, we have

$$
\begin{aligned}
\alpha(S X) & =\sum_{i} \alpha\left(e_{i}\right) g\left(S e_{i}, X\right)=\sum_{i} g\left(\left(\nabla_{e_{i}} S\right) e_{i}, X\right) \\
& =\frac{1}{2} X r=\frac{1}{2} \sum_{i} X g\left(S e_{i}, e_{i}\right)=\frac{1}{2} \sum_{i} g\left(\left(\nabla_{X} S\right) e_{i}, e_{i}\right) \\
& =\frac{1}{2} \alpha(X) r
\end{aligned}
$$

where the third equality is given by the second Bianchi identity. That is, we have the following

$$
\alpha(X)\left\{g\left(S^{2} Z, Y\right)-\frac{1}{2} r g(S Z, Y)\right\}+\alpha(Z)\left\{g\left(S^{2} X, Y\right)-\frac{1}{2} r g(S X, Y)\right\}=0 .
$$

If $\alpha(X) \neq 0$, setting $X=Z$, we have $S^{2}=(r / 2) S$. If $\alpha(X)=0$, taking $Z$ such that $\alpha(Z) \neq 0, S^{2}=(r / 2) S$. Consequently we have $S^{2}=(r / 2) S$.

In the proof of Proposition 4.1, we have

LEMMA 4.2. Let $M$ be a Ricci recurrent manifold of dimension $n$. Then the curvature tensor $R$ and the Ricci tensor $S$ satisfy $R(X, Y) S=0$ for any vector fields $X$ and $Y$.

Lemma 4.2 gives the relation between Ricci recurrent condition and Ricci semi-symmetry.

REMARK 4.3. From Lemma 4.2 and a theorem of [5], we see that there are no real hypersurfaces with recurrent Ricci tensor of $M^{m}(c), m \geq 3$, (Loo [8]).

THEOREM 4.4. Let $M$ be an n-dimensional $C R$ submanifold of a complex space form $M^{m}(c), c \neq 0$, with semi-flat normal connection. If $\operatorname{dim} H_{x}>2$, then $M$ is not Ricci recurrent. 
Proof. We suppose that $M$ is a Ricci recurrent $C R$ submanifold of $M^{m}(c)$, $h>2$, with semi-flat normal connection. Since $S$ is symmetric, by Theorem 3.1, we can choose an orthonormal basis $\left\{e_{1}, \ldots, e_{h}, t v_{1}, \ldots, t v_{q}\right\}$ of $T_{x}(M)$ such that the Ricci tensor $S$ is represented by a matrix form

$$
S=\left(\begin{array}{cccccc}
a & \cdots & 0 & & & \\
\vdots & \ddots & \vdots & & * & \\
0 & \cdots & a & & & \\
& & & \lambda_{1} & \cdots & 0 \\
& * & & \vdots & \ddots & \vdots \\
& & & 0 & \cdots & \lambda_{p}
\end{array}\right)
$$

where we have put

$$
a=\frac{1}{h}\left(r-\sum_{a} g\left(S t v_{a}, t v_{a}\right)\right) .
$$

By Lemma 3.1, we see that eigenvalues of $S$ are $r / 2$ and 0 , and that $\operatorname{rank} S=2$. Since $h>2$, we can assume that $S$ is represented by a matrix form

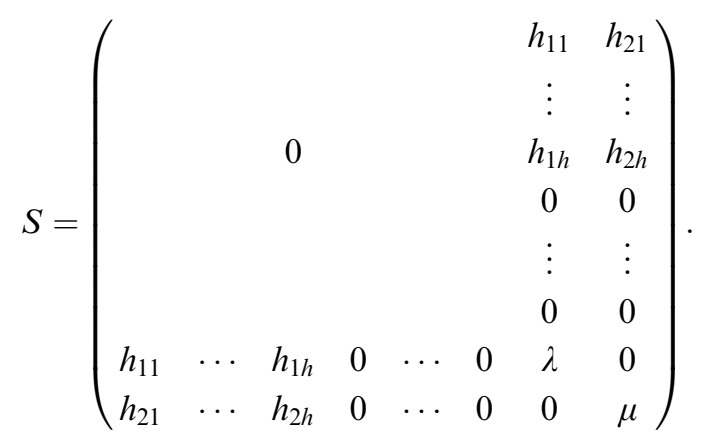

Thus we have

$$
\begin{aligned}
& \operatorname{tr} S^{2}=\lambda^{2}+\mu^{2}+\sum_{i=1}^{h} h_{1 i}^{2}+\sum_{i=1}^{h} h_{2 i}^{2}, \\
& \operatorname{tr}\left(\frac{r}{2} S\right)=\frac{1}{2}(\lambda+\mu)^{2} .
\end{aligned}
$$

Since $S^{2}=(r / 2) S$, we have $\lambda=\mu=r / 2, h_{1 i}=0$ and $h_{2 i}=0$ for $i=1, \ldots, h$. Thus we see that $\{X \in T M \mid S X=(r / 2) X\} \subset H_{x}^{\perp}$. Then there is a vector field 
$v \in J H_{x}^{\perp}$ such that $S t v=(r / 2) t v$. We notice that $J v=t v \in H_{x}$ and $f v=0$. We obtain

$$
\left(\nabla_{X} S\right) t v+S \nabla_{X} t v=\frac{1}{2}(X r) t v+\frac{r}{2} \nabla_{X} t v
$$

On the other hand, in the proof of Proposition 3.1, we have $X r=\alpha(X) r$. Then

$$
\left(\nabla_{X} S\right) t v=\alpha(X) S t v=\frac{r}{2} \alpha(X) t v=\frac{1}{2}(X r) t v .
$$

So we obtain

$$
S \nabla_{X} t v=\frac{r}{2} \nabla_{X} t v
$$

Thus we see that $\nabla_{X} t v \in H_{x}^{\perp}$. From the equations $\nabla_{X} t v-t D_{X} v=\left(\nabla_{X} t\right) v=$ $-P A_{v} X+A_{f v} X$ and $f v=0$, we see that $\nabla_{X} t v-t D_{X} v=-P A_{v} X$. Since the lefthand side is in $H_{x}^{\perp}$ and the right-hand side is in $H_{x}$, we have $\nabla_{X} t v=t D_{X} v$. So we obtain

$$
\begin{aligned}
& \nabla_{Y} \nabla_{X} t v=\nabla_{Y}\left(t D_{X} v\right)=t D_{Y} D_{X} v \\
& \nabla_{X} \nabla_{Y} t v=\nabla_{X}\left(t D_{Y} v\right)=t D_{X} D_{Y} v \\
& \nabla_{[X, Y]} t v=t D_{[X, Y]} v .
\end{aligned}
$$

Since the normal connection of $M$ is semi-flat, we have

$$
R(X, Y) t v=t R^{\perp}(X, Y) v=2 c g(X, P Y) t f v=0 .
$$

By the definition of the Ricci tensor $S$, we see

$$
\frac{r}{2}=g(S t v, t v)=\sum_{i} g\left(R\left(e_{i}, t v\right) t v, e_{i}\right)=0 .
$$

So we have $S=0$. This is a contradiction.

From Theorem 4.4, we have the following theorem about generic submanifold.

THEOREM 4.5. Let $M$ be an n-dimensional generic submanifold of a complex space form $M^{m}(c), c \neq 0$, with flat normal connection. If $n-p>2$, then $M$ is not Ricci recurrent. 


\section{A Characterization of Pseudo-Einstein Real Hypersurfaces}

In this section, we give a characterization of pseudo-Einstein real hypersurfaces of a complex space form by using Corollary 3.3.

Let $M$ be a real $(2 m-1)$-dimensional hypersurface immersed in a complex space form $M^{m}(c)$. We take the unit normal vector field $N$ of $M$ in $M^{m}(c)$. For any vector field $X$ tangent to $M$, we define $P, \eta$ and $\xi$ by

$$
J X=P X+\eta(X) N, \quad \xi=-J N,
$$

where $P X$ is the tangential part of $J X, P$ is a tensor field of type $(1,1), \eta$ is a 1 -form, and $\xi$ is the unit vector field on $M$. Then they satisfy

$$
P^{2} X=-X+\eta(X) \xi, \quad P \xi=0, \quad \eta(P X)=0
$$

for any vector field $X$ tangent to $M$. Moreover, we have

$$
\begin{gathered}
g(P X, Y)+g(X, P Y)=0, \quad \eta(X)=g(X, \xi), \\
g(P X, P Y)=g(X, Y)-\eta(X) \eta(Y) .
\end{gathered}
$$

Thus $(P, \xi, \eta, g)$ defines an almost contact metric structure on $M$.

The Gauss and Weingarten formulas are given respectively by

$$
\tilde{\nabla}_{X} Y=\nabla_{X} Y+g(A X, Y) N, \quad \tilde{\nabla}_{X} N=-A X
$$

for any vector fields $X$ and $Y$ tangent to $M$. We call $A$ the shape operator (second fundamental form) of $M$.

For the contact metric structure on $M$ we have

$$
\nabla_{X} \xi=P A X, \quad\left(\nabla_{X} P\right) Y=\eta(Y) A X-g(A X, Y) \xi .
$$

The equation of Gauss is given by

$$
\begin{aligned}
R(X, Y) Z=c\{ & g(Y, Z) X-g(X, Z) Y+g(P Y, Z) P X \\
& -g(P X, Z) P Y-2 g(P X, Y) P Z\}+g(A Y, Z) A X-g(A X, Z) A Y .
\end{aligned}
$$

By the equation of Gauss, the Ricci tensor $S$ of type $(1,1)$ of $M$ is given by

$$
S X=(2 n+1) c X-3 c \eta(X) \xi+h A X-A^{2} X,
$$

where $h$ denotes the mean curvature of $M$ given by the trace of the shape operator $A$. Moreover, the scalar curvature $r$ of $M$ is given by

$$
r=4\left(n^{2}-1\right) c+h^{2}-\operatorname{Tr} A^{2} .
$$


If the Ricci tensor $S$ of $M$ is of the form $g(S X, Y)=a g(X, Y)+b \eta(X) \eta(Y)$ for some functions $a$ and $b$, then $M$ is said to be pseudo-Einstein. Then $a$ and $b$ are constant when $m \geq 3$.

THEOREM 5.1. Let $M$ be a real hypersurface of a complex space form $M^{m}(c)$, $c \neq 0, m \geq 3$. Then the curvature tensor $R$ and the Ricci tensor $S$ of $M$ satisfy $g((R(X, Y) S) Z, W)=0$ for any tangent vector fields $X, Y, Z$ and $W$ orthogonal to $\xi$ if and only if $M$ is pseudo-Einstein.

Proof. We suppose that $M$ satisfies $g((R(X, Y) S) Z, W)=0$ for any tangent vector fields $X, Y, Z$ and $W$ orthogonal to $\xi$. We can choose an orthonormal basis $\left\{X_{1}, \ldots, X_{2 m-2}, \xi\right\}$ of $M$ such that the shape operator $A$ is represented by a matrix form

$$
A=\left(\begin{array}{cccc}
\lambda_{1} & \cdots & 0 & h_{1} \\
\vdots & \ddots & \vdots & \vdots \\
0 & \cdots & \lambda_{2 m-2} & h_{2 m-2} \\
h_{1} & \cdots & h_{2 m-2} & \alpha
\end{array}\right)
$$

Then, we have

$$
\begin{aligned}
S X_{i} & =(2 n+1) c X_{i}-3 c \eta\left(X_{i}\right) \xi+h A X_{i}-A^{2} X_{i} \\
& =\left((2 n+1) c+h \lambda_{i}-\lambda_{i}^{2}\right) X_{i}+h_{i}\left(h-\lambda_{i}-\alpha\right) \xi-\sum_{k=1}^{2 m-2} h_{i} h_{k} X_{k}, \\
S \xi & =(2 m+1) c \xi-3 c \eta(\xi) \xi+h A \xi-A^{2} \xi \\
& =(2 m-2) c \xi+h\left(\sum_{k=1}^{2 m-2} h_{k} X_{k}+\alpha \xi\right)-A\left(\sum_{k=1}^{2 m-2} h_{k} X_{k}+\alpha \xi\right) \\
& =\sum_{k=1}^{2 m-2} h_{k}\left(h-\lambda_{k}-\alpha\right) X_{k}+\left((2 m-2) c+\alpha h-\sum_{k=1}^{2 m-2} h_{k}^{2}-\alpha^{2}\right) \xi .
\end{aligned}
$$

By Corollary 3.3, we have

$$
\begin{aligned}
& g\left(S X_{i}, X_{j}\right)=-h_{i} h_{j}=0 \quad(i \neq j), \\
& g\left(S X_{i}, X_{i}\right)=\frac{1}{2 n-2}(r-g(S \xi, \xi)) \quad(i=1, \ldots, 2 m-2) .
\end{aligned}
$$


Equation (5.1) shows that at most one $h_{i}$ does not vanish. Thus we can assume that $h_{i}=0$ for $i=2, \ldots, 2 m-2$. We set $a=g\left(S X_{i}, X_{i}\right)$. Then we have

$$
\begin{aligned}
& S X_{1}=a X_{1}+h_{1}\left(h-\lambda_{1}-\alpha\right) \xi \\
& S X_{i}=a X_{i} \quad(i=2, \ldots, 2 n-2), \\
& S \xi=h_{1}\left(h-\lambda_{1}-\alpha\right) X_{1}+\left((2 m-2) c+\alpha h-h_{1}^{2}-\alpha^{2}\right) \xi .
\end{aligned}
$$

Since $g((R(X, Y) S) Z, W)=0$ for any tangent vector fields $X, Y, Z$ and $W$ orthogonal to $\xi$, we have

$$
g(R(X, Y) S Z-S R(X, Y) Z, W)=0 .
$$

By the equation of Gauss, for any $j \geq 2$, we obtain

$$
\begin{aligned}
0 & =g\left(R\left(X_{1}, X_{j}\right) S X_{1}, X_{j}\right)-g\left(S R\left(X_{1}, X_{j}\right) X_{1}, X_{j}\right) \\
& =a g\left(R\left(X_{1}, X_{j}\right) X_{1}, X_{j}\right)+h_{1}\left(h-\lambda_{1}-\alpha\right) g\left(R\left(X_{1}, X_{j}\right) \xi, X_{j}\right)-\operatorname{ag}\left(R\left(X_{1}, X_{j}\right) X_{1}, X_{j}\right) \\
& =h_{1}\left(h-\lambda_{1}-\alpha\right) g\left(R\left(X_{1}, X_{j}\right) \xi, X_{j}\right) .
\end{aligned}
$$

By the equation of Gauss, we have

$$
\begin{aligned}
g\left(R\left(X_{1}, X_{j}\right) \xi, X_{j}\right) & =g\left(A X_{j}, \xi\right) g\left(A X_{1}, X_{j}\right)-g\left(A X_{1}, \xi\right) g\left(A X_{j}, X_{j}\right) \\
& =-h_{1} \lambda_{j} .
\end{aligned}
$$

Thus we see that $h_{1}^{2} \lambda_{j}\left(h-\lambda_{1}-\alpha\right)=0$ for $j \geq 2$. If $h_{1}\left(h-\lambda_{1}-\alpha\right) \neq 0$, then we have $\lambda_{j}=0$ for $j \geq 2$. Since $h=\operatorname{Tr} A$, we have $h=\lambda_{1}+\alpha$. This is a contradiction. So we have $h_{1}\left(h-\lambda_{1}-\alpha\right)=0$. By (5.3), we see that $M$ is pseudoEinstein and that $h_{1}=0$ (see [7]). Thus we see that, if $g((R(X, Y) S) Z, W)=0$ for any tangent vector fields $X, Y, Z$ and $W$ orthogonal to $\xi$, then $M$ is pseudoEinstein.

Conversely, if $M$ is pseudo-Einstein, we have $S Z=a Z+b \eta(Z) \xi=a Z$ and $S W=a W$ for any tangent vectors $Z$ and $W$ orthogonal to $\xi$. Then we have $g((R(X, Y) S) Z, W)=g(R(X, Y) S Z, W)-g(S R(X, Y) Z, W)=0$.

We need the following two theorems of pseudo-Einstein real hypersurfaces in a complex projective space $C P^{m}$ with constant holomorphic sectional curvature 4 (Cecil and Ryan [1], Kon [7]) and a complex hyperbolic space $\mathrm{CH}^{m}$ with constant holomorphic sectional curvature -4 (Montiel [9]). 
THEOREM A. Let $M$ be a complete and connected real hypersurface in $C P^{m}$, $m \geq 3$, which is pseudo-Einstein. Then $M$ is congruent to one of the following spaces:

(a) a geodesic hypersphere,

(b) a tube of radius $r$ over a totally geodesic $C P^{k}, 0<k<m-1$, where $0<r<\pi / 2$ and $\cot ^{2} r=k /(m-k-1)$,

(c) a tube of radius $\pi / 4-\theta$ over a complex quadric $Q^{m-1}$ where $0<\theta<\pi / 4$ and $\cot ^{2} 2 r=m-2$.

THEOREM B. Let $M$ be a complete and connected real hypersurface of $\mathrm{CH}^{m}$, $m \geq 3$, which is pseudo-Einstein. Then $M$ is congruent to one of the following spaces:

(a) a geodesic hypersphere.

(b) a tube of radius $r>0$ over a complex hyperbolic hyperplane $\mathrm{CH}^{m-1}$.

(c) a self-tube $M_{m}^{*}$.

Using Theorem A and Theorem B, Theorem 5.1 implies the following theorems.

THEOREM 5.2. Let $M$ be a complete and connected real hypersurface of $C P^{m}, m \geq 3$. Suppose that the curvature tensor $R$ and the Ricci tensor $S$ satisfy $g((R(X, Y) S) Z, W)=0$ for any tangent vector fields $X, Y, Z$ and $W$ orthogonal to $\xi$. Then $M$ is congruent to one of the following spaces:

(a) a geodesic hypersphere,

(b) a tube of radius $\theta$ over a totally geodesic $C P^{k}, 0<k<m-1$, where $0<\theta<\pi / 2$ and $\cot ^{2} \theta=k /(m-k-1)$,

(c) a tube of radius $\pi / 4-\theta$ over a complex quadric $Q^{m-1}$ where $0<\theta<\pi / 4$ and $\cot ^{2} 2 \theta=m-2$.

THEOREM 5.3. Let $M$ be a complete and connected real hypersurface of $C H^{m}, m \geq 3$. Suppose that the curvature tensor $R$ and the Ricci tensor $S$ satisfy $g((R(X, Y) S) Z, W)=0$ for any tangent vector fields $X, Y, Z$ and $W$ orthogonal to $\xi$. Then $M$ is congruent to one of the following spaces:

(a) a geodesic hypersphere $M_{0, m-1}^{h}\left(\tanh ^{2} \theta\right)$ of radius $r>0$,

(b) a tube $M_{m-1,0}^{h}\left(\tanh ^{2} \theta\right)$ of radius $\theta>0$ over a complex hyperbolic hyperplane,

(c) a self-tube $M_{m}^{*}$. 
As an application of Theorem 5.1, we prove the following theorem (see [5], $[6])$.

THEOREM 5.4. There are no real hypersurfaces with $R(X, Y) S=0$, semisymmetric Ricci tensor, of a complex space form $M^{m}(c), c \neq 0, m \geq 3$.

Proof. We suppose that the Ricci tensor $S$ of the real hypersurface $M$ is semi-symmetric, that is, the curvature tensor and the Ricci tensor satisfy $R(X, Y) S=0$ for any tangent vector fields $X$ and $Y$. Then by Theorem 5.1, the real hypersurface $M$ is pseudo-Einstein. Consequently, the Ricci tensor $S$ satisfies $S X_{i}=a X_{i}$ for $i=1, \ldots, 2 m-2$ and $S \xi=\left(c(2 n-2)+\alpha h-\alpha^{2}\right) \xi:=b \xi$. Then, for any $i=1, \ldots, 2 m-2$, we have

$$
\begin{aligned}
0 & =R\left(\xi, X_{i}\right) S \xi-S R\left(\xi, X_{i}\right) \xi \\
& =b R\left(\xi, X_{i}\right) \xi-S R\left(\xi, X_{i}\right) \xi \\
& =b\left\{-c g(\xi, \xi) X_{i}-g(A \xi, \xi) A X_{i}\right\}-S\left\{-c g(\xi, \xi) X_{i}-g(A \xi, \xi) A X_{i}\right\} \\
& =-b c X_{i}-b \alpha \lambda_{i} X_{i}+a c X_{i}+a \alpha \lambda_{i} X_{i} \\
& =(a-b)\left(c+\alpha \lambda_{i}\right) X_{i}
\end{aligned}
$$

Since $b \neq a$, we have $\lambda_{i}=-c / \alpha, i=1, \ldots, 2 m-2$. We put $\lambda=-c / \alpha$. Suppose that $X$ is a unit vector field orthogonal to $\xi$. Then we have

$$
\begin{aligned}
\nabla_{X} \nabla_{\xi} \xi & =\nabla_{X} P A \xi=0 \\
\nabla_{\xi} \nabla_{X} \xi & =\nabla_{\xi} P A X=\lambda \nabla_{\xi} P X \\
& =\lambda\left(\nabla_{\xi} P\right) X+\lambda P \nabla_{\xi} X \\
& =\lambda(\eta(X) A \xi-g(A \xi, X) \xi)+\lambda P \nabla_{\xi} X \\
& =\lambda P \nabla_{\xi} X \\
\nabla_{[X, \xi]} \xi & =P A[X, \xi] \\
& =P A \nabla_{X} \xi-P A \nabla_{\xi} X \\
& =P A P A X-P A \nabla_{\xi} X \\
& =\lambda^{2} P^{2} X-P A \nabla_{\xi} X \\
& =-\lambda^{2} X-P A \nabla_{\xi} X .
\end{aligned}
$$


Thus we obtain

$$
\begin{aligned}
R(X, \xi) \xi & =\nabla_{X} \nabla_{\xi} \xi-\nabla_{\xi} \nabla_{X} \xi-\nabla_{[X, \xi]} \xi \\
& =-\lambda P \nabla_{\xi} X+\lambda^{2} X+P A \nabla_{\xi} X
\end{aligned}
$$

So we have

$$
\begin{aligned}
g(R(X, \xi) \xi, X) & =-\lambda g\left(P \nabla_{\xi} X, X\right)+\lambda^{2} g(X, X)+g\left(P A \nabla_{\xi} X, X\right) \\
& =\lambda g\left(\nabla_{\xi} X, P X\right)+\lambda^{2} g(X, X)-\lambda g\left(\nabla_{\xi} X, P X\right) \\
& =\lambda^{2} g(X, X)=\lambda^{2}
\end{aligned}
$$

By the equation of Gauss, we have $g(R(X, \xi) \xi, X)=c+\alpha \lambda=0$. These equations imply $\lambda=0$ and $c=0$. This is a contradiction. So we have our theorem.

REMARK 5.5. We can see that the totally $\eta$-umbilical pseudo-Einstein real hypersurfaces of $C P^{m}$ and $C H^{m}$ satisfies $c+\alpha \lambda \neq 0$ by a straightforward computation using principal curvatures of examples (see [6]). Here, we proved Theorem 5.4 by a slight general method.

\section{References}

[ 1 ] T. E. Cecil and P. J. Ryan, Focal sets and real hypersurfaces in complex projective space, Trans. Amer. Math. Soc. 269 (1982), 481-499.

[2] B. Y. Chen, Geometry of submanifolds, Marcel Dekken Inc., New York, 1973.

[3] T. Hamada, On real hypersurfaces of a complex projective space with recurrent Ricci tensor, Glasgow Math. J. 41 (1999), 297-302.

[ 4 ] U-Hang Ki, Real hypersurfaces with parallel Ricci tensor of a complex space form, Tsukuba $\mathbf{J}$. Math. 13 (1989), 73-81.

[ 5 ] U-Hang Ki, H. Nakagawa and Y. J. Suh, Real hypersurfaces with harmonic Weyl tensor of a complex space form, Hiroshima Math. J. 20 (1990), 93-102.

[6] M. Kimura and S. Maeda, On real hypersurfaces of a complex projective space III, Hokkaido Math. J. 22 (1993), 63-78.

[ 7 ] M. Kon, Pseudo-Einstein real hypersurfaces in complex space forms, J. Differential Geom. 14 (1979), 339-354.

[ 8 ] T-H. Loo, Real hypersurfaces in a complex space form with recurrent Ricci tensor, Glasgow Math. J. 44 (2002), 547-550.

[ 9 ] S. Montiel, Real hypersurfaces of a complex hyperbolic space, J. Math. Soc. Japan 37 (1985), 515-535.

[10] Z. Olszak, On Ricci recurrent manifolds, Coll. Math. 52 (1987), 205-211.

[11] E. M. Patterson, On symmetric recurrent tensors of the second order, Quart. J. Math., Oxford Ser. 2 (1951), 151-158.

[12] E. M. Patterson, Some theorems on Ricci recurrent spaces, J. London. Math. Soc, 27 (1952), 287-295.

[13] W. Roter, Some remarks on infinitesimal projective transformations in recurrent and Ricci recurrent spaces, Coll. Math. 15 (1966), 121-127. 
[14] K. Yano, On a structure defined by a tensor field $f$ of type $(1,1)$ satisfying $f^{3}+f=0$, Tensor N. S. 14 (1963), 99-109.

[15] K. Yano and M. Kon, Structures on manifolds, World Scientific Publishing, Singapore, 1984.

Department of Mathematics

Hokkaido University

Kita 10 Nishi 8

Sapporo 060-0810, Japan

E-mail address: mayuko_k13@math.sci.hokudai.ac.jp 